\title{
LA SIMULATION DE L'ACTE D'ÉNONCIATION : LE CAS DE LAURENT BINET
}

\author{
Veronika ČERNÍKOVÁ \\ Université de Bohême du Sud à České Budějovice
}

\begin{abstract}
En): The paper presents a reflection on John Searle's theory according to whom the fiction pretends illocutionary acts whereas the utterance act remains real. Yet we suppose that the meta-novels pretend also the utterance act which no longer represents the only truthful act of fiction but rather a literary convention. To confirm our hypothesis, we chose to analyse $H H h H$ by Laurent Binet, a contemporary French author. His novel lends itself well to the analysis because the author plays with the reliability of the narrator which is not as credible as it may seem; with the reader who remains abandoned in the maze built from the pieces of reality and fiction, unable to distinguish one from the other; and especially with the nature of the fictional world which, deprived of authentication, remains virtual, inhabited by phantoms floating somewhere between being and the non-being. The intentionality of the author is all the more obvious as the second novel of Binet, The $7^{\text {th }}$ Function of Language, is perfectly antithetical to the first one. This systematic work of the author shows that his sincerity is not sincere, it is actually consciously fabricated and with it the utterance act. But if the utterance act is constructed, if it is not sincere, isn't it also pretended?
\end{abstract}

Keywords (En): Binet; meta-novel ; lie truly ; reliable narrator ; fictionality ; illusion of the reality

Mots-clés (Fr): Binet ; méta-roman ; mentir vrai ; narrateur fiable ; fictionnalité ; illusion de la réalité

Dans son article sur le statut logique du discours fictionnel, John Searle explique que chaque fiction prétend, simule ou imite les actes illocutoires. Elle ne le fait pas pour tromper le lecteur: il ne s'agit pas de mensonge mais d'une pseudo-performance non trompeuse (nondeceptive pseudoperformance) à caractère ludique. C'est grâce à l'existence des conventions littéraires que l'auteur peut simuler la réalisation d'une série d'actes illocutoires, ce qui distingue effectivement la fiction du mensonge. Si l'acte illocutoire est simulé, l'acte d'énonciation est réel. (SEARLE, 2007 : 65) Néanmoins, dans certains romans, dont le nombre va croissant, l'acte d'énonciation peut être également simulé. Pensons aux méta-romans dont le narrateur se déguise en auteur empirique du roman que nous sommes en train de lire, il s'adresse directement à ses lecteurs et commente son écriture. Le récit est non seulement conscient de lui-même, il impose sa fictionnalité au lecteur. En effet, depuis Gide pour qui l'acte d'écriture (et avec lui l'acte d'énonciation) est le seul fait véridique, la pratique novatrice s'est transformée en un lieu commun, elle est devenue une convention littéraire. De ce fait, il faut se poser la question si une telle pratique peut toujours être liée à la véridicité. N'est-elle pas plutôt au service de la mimèsis, ne devient-elle pas qu'un nouveau trompe-l'œil dont l'auteur se sert pour construire l'illusion de la réalité ? D'où notre hypothèse que, devenue convention littéraire, la méta-écriture permet à l'auteur de simuler non seulement les actes illocutoires, mais aussi ledit acte d'énonciation, tout cela dans un régime ludique, c'est-à-dire sans avoir 
nécessairement recours au mensonge. Dans le texte qui suit, nous tenterons d'appuyer cette hypothèse par l'analyse de $H H h H(2009)^{1}$, le premier roman de Laurent Binet, ainsi que par plusieurs brèves comparaisons avec son deuxième roman, La septième fonction du langage (2015).

Le choix de l'auteur est doublement motivé. D'une part, il s'agit d'un représentant idéal de la littérature française contemporaine qui sait mélanger les genres tout en combinant ceux qui sont les plus populaires : le roman historique au roman autobiographique, au polar et à beaucoup d'autres. À l'instar de son grand maître Umberto $\mathrm{Eco}^{2}$, il écrit des romans complexes mais qui restent accessibles au grand public grâce à leur lisibilité et leur suspense. D'autre part, Binet est un auteur érudit qui connaît bien le milieu au sujet duquel il écrit. Il a fait son service militaire en Slovaquie et, pendant plusieurs années, il a partagé sa vie entre Paris et Prague où il rassemblait la documentation pour son premier roman, dont la rédaction lui a pris dix ans (STRANSKY, 2014). Comme le protagoniste de La septième fonction du langage, il a été chargé de cours en sémiologie à Paris 8 (exVincennes). C'est d'ailleurs à cette université que son père avait étudié la géographie. (LEVISALLES, 2015) Binet lui-même souligne que son deuxième roman est l'œuvre de «recherches monumentales» qui ont duré cinq ans (SARFATI, 2015). Pendant ce temps, il a voyagé, interviewé des témoins, étudié les textes des auteurs qu'il avait décidé de transformer en personnages (LEVISALLES, 2015). Mais surtout, Binet est très bien conscient de ce qu'il fait et pourquoi il le fait. Il prétend que la littérature est entièrement une question de forme et que, pour être intéressant, un roman doit être également un méta-roman (STRANSKY, 2014). Et effectivement, ses romans représentent deux variantes de méta-roman assez divergentes.

À première vue, $H H h H$ est un roman historique focalisé sur « un des plus grands actes de résistance de l'histoire humaine » (p. 10) : l'opération Anthropoïde qui a pour but l'élimination de Reinhard Heydrich, la bête blonde, le bourreau de Prague, le planificateur de la Solution finale. Successivement, nous suivons l'enfance d'Heydrich, son adolescence, sa montée en grade jusqu'à la position de l'homme le plus dangereux du Reich. Nous assistons parallèlement à la montée du nazisme, à l'Anschluss d'Autriche, à l'annexion des Sudètes, à l'entrainement des parachutistes, aux préparatifs de l'attentat. Tous les chemins de la narration tendent vers le moment le plus dramatique qui scelle le destin de centaines de personnes et sauve probablement la vie de milliers. L'intrigue historique s'amalgame avec les procédés empruntés aux thrillers, polars et romans d'espionnage pour créer un roman palpitant. Néanmoins, cette guerre entre les forces du bien et du mal en cache une autre, non moins dramatique, celle que livre la fiction romanesque à la vérité historique. Et c'est précisément cette deuxième

\footnotetext{
${ }^{1}$ Toutes les références à $H H h H$, ainsi que les citations, proviennent de l'édition BINET Laurent (2009), HHhH, Paris, Grasset.

2 Dans son article «Teasing the French academy to death», Nicholas LEZARD montre bien que La septième fonction du langage doit beaucoup au Nom de la Rose d'Umberto Eco qui a plus ou moins inventé le thriller au concept intellectuel actualisant la méthodologie de Sherlock Holmes (2017). Binet lui-même déclare qu'en élaborant l'intrigue, il a pensé sans s'en rendre compte «à un pitch à la Umberto Eco : un manuscrit que tout le monde convoite et pour lequel on tue. » (PHILIPPE 2015b)
} 
lutte qui nourrit la dimension méta-romanesque de $H H h H$ qui est, de fait, surtout un roman sur la création difficile d'un roman historique, une reconstruction de l'enquête et des recherches qui aboutissent à cette création. Cela permet au lecteur de suivre chronologiquement non seulement les préparatifs et la réalisation de l'opération mais aussi les préparatifs et la rédaction du roman qu'il est en train de lire. En outre, Binet décide d'associer la figure du narrateur à celle de l'auteur ${ }^{3}$ ce qui lui permet de s'adresser directement au lecteur, de lui expliquer son comportement et de lui indiquer, de manière obsessionnelle, la part de l'invention.

La septième fonction du langage est souvent jugée un roman inclassable. En cherchant une bonne étiquette générique, certains critiques optent pour celle du polar érudit (LEMENAGER, 2015), sémiologique (BORDERIE, 2015), historique (BIRNBAUM, 2015) ou politique (SAMOYAULT, 2015); il est possible de repérer également la notion de «méta-polar » (PHILIPPE, 2015a). Certains, notamment des critiques américains, parlent plutôt de thriller et se trouve parmi eux aussi un Français qui précise qu'il s'agit d'un « gros thriller ésotérico-complotiste à la manière de Dan Brown » (LIGER, 2015). Plusieurs associent le roman à la farce, philosophique (BIRNBAUM, 2015) ou autre. Laurent Binet le qualifie comme un roman baroque «qui se décrit justement par le mélange des genres » (SARFATI, 2015) :

J'aimais bien l'idée que mon livre soit un roman populaire et un roman savant, un roman policier et un road-movie, un méta-roman et un roman picaresque, un roman d'aventures et un roman d'espionnage à la James Bond, un roman comique mais également épique, et qu'il y ait quelque chose de l'ordre du western italien. (HAMOUCHI, 2015)

Ces différents classements montrent bien les domaines d'intérêt de l'auteur: l'intrigue fondée sur l'action (l'enquête de la mort de Roland Barthes et la quête de la superpuissante septième fonction du langage), ancrée dans un milieu intellectuel (la French Theory) et se déroulant sur un fond historico-politique (la veille de l'élection présidentielle de 1981). Contrairement à $H H h H$, la composante méta-romanesque n'est pas si évidente ni si étendue bien qu'elle n'en soit pas moins importante. Encore une fois, Binet n'hésite pas à introduire la figure de l'auteur qui, pourtant, ne s'adresse au lecteur que rarement. Il paraît que cette fonction est reprise par le protagoniste Simon Herzog. Celui-ci apostrophe l'auteur, est conscient de son statut ontologique, refuse d'être marionnettisé et paraît usurper la fonction performative du narrateur, notamment grâce à sa capacité extraordinaire de faire des analyses sémiologiques qui le rapprochent de Sherlock Holmes, dont il porte les initiales, mais aussi parce qu'il est capable de décider du dénouement du roman. Ne connaît-il pas finalement le secret de la septième fonction du langage ? En outre, Binet introduit comme personnages les

3 Binet n'aime pas distinguer entre l'auteur et le narrateur : «The distinction between author and narrator is usually artificial, or not very relevant. In many books the narrator is not the author, but I think it's useless to invent a narrator who's just a mouthpiece for the author. The problem is, at least in France, many people believe that it's a code for literature: If you don't have a narrator you aren't writing literature. Writers believe that they have to invent a narrator even if it doesn't add anything to the story. It's better, in my case and in many other cases, to just skip that whole operation. » (STRANSKY, 2014) 
grands théoriciens de la fiction (Roland Barthes, Michel Foucault, Umberto Eco, John Searle et beaucoup d'autres) tout en décidant de faire « diverger [son] roman de l'histoire officielle » (SARFATI, 2015). Un peu à la Derrida, son roman «sape lui-même ses propres bases. Personne ne va croire que Barthes a été vraiment assassiné. » (PHILIPPE, 2015b) Pour en être sûr, Binet introduit un nombre élevé d'éléments subversifs qui doivent perturber l'illusion de la réalité. Outre les services secrets de plusieurs pays et d'autres motifs manifestement imaginaires, l'auteur introduit aussi des lézardes moins évidentes mais d'autant plus inquiétantes comme la présence de Morris Zapp, professeur de Changing places de David Lodge, ou la chanson Kids in America qui représente un léger anachronisme (TREINER, 2015). D'après ses propres mots, il veut mettre en relief « l'ambition fictionnelle qui est de toujours un peu supplanter le réel. » (SARFATI, 2015) Le principe de ce méta-roman est alors d'exhiber sa fictionnalité et, dans son ensemble, de réfléchir non seulement sur lui-même mais aussi sur le roman en général.

Bien que les deux méta-romans de Laurent Binet représentent des projets différents, ils partagent la thématisation de la relation problématique entre la réalité et la fiction. Binet s'exprime sur ce point assez clairement: «Ce que je n'aime pas, c'est quand on fait comme si ce rapport n'était pas problématique. Soit on raconte une histoire vraie en s'attardant à la réalité des faits, soit on exhibe ce rapport problématique. Entre les deux, ça ne m'intéresse pas tellement. » (SARFATI, 2015) Ainsi, dans $H H h H$, Binet est obsédé par la vérité historique sans vouloir abandonner entièrement le genre romanesque. Mais comment écrire un roman historique qui aspirerait à la véracité ? Pour authentifier son récit, Binet choisit une stratégie narrative qui n'est pas des plus évidentes : le narrateur fiable.

Pour étudier le rôle du narrateur fiable dans $H H h H$, il nous paraît utile de nous arrêter brièvement sur la théorie des mondes possibles présentée par Lubomír DOLEŽEL dans Heterocosmica. En écrivant un texte fictionnel, l'écrivain construit un monde fictionnel ${ }^{4}$ par l'intermédiaire de l'énonciation du narrateur et/ou des personnages. (2003 : 37) Comme cette énonciation a une fonction constructive, elle peut être comparée à l'acte performatif, défini par J. L. AUSTIN comme une énonciation qui transforme les mots en action : " the issuing of the utterance is the performing of an action» (1962: 6). L'énonciation du narrateur signe l'acte de naissance du monde fictionnel. Comme les textes fictionnels représentent des assemblages de performatifs, ils n'obéissent pas à l'évaluation de la véracité. Si nous voulons vérifier ce qui existe dans un monde fictionnel et ce qui n'y existe pas, il faut discerner ce qui est authentifié de ce qui ne l'est pas. (DOLEŽEL, 2003 : 149-150) DOLEŽEL distingue plusieurs degrés d'authentification d'après les types de narrateur. Le narrateur objectif du type balzacien dont l'acte narratif est un acte performatif comparable au Verbe de Dieu est doté d'une autorité performative absolue : tous les motifs qu'il introduit dans son discours sont authentifiés et, de ce fait, dotés de l'existence fictionnelle. (2003: 151-5) Par contre, le narrateur

\footnotetext{
${ }^{4}$ Nous tenons à souligner que, contrairement à Searle, Doležel ne distingue pas la fiction et la littérature. Pour lui chaque texte littéraire est un texte fictionnel. De ce fait, un monde fictionnel ne peut jamais être identique au monde actuel.
} 
personnel dispose d'une autorité performative limitée qui ne lui est pas donnée $a$ priori: soit il s'approprie l'autorité performative du narrateur objectif, soit il s'efforce de la mériter en se comportant comme un témoin fiable. (2003 : 155-161) Pour authentifier son monde fictionnel, Laurent Binet choisit cette troisième possibilité qui est pourtant la moins évidente car l'autorité performative d'un tel narrateur est la plus faible et la moins stable. Son narrateur personnel doit littéralement lutter tout au long du roman pour être cru. Dans le texte qui suit, nous tenterons de vérifier s'il mérite vraiment de l'être, s'il respecte les cinq piliers sur lesquels repose, au moins d'après DOLEŽEL, la crédibilité du narrateur jouant le rôle du témoin fiable (2003: 157-8) :

1) ne parler que des motifs qu'il connaît

2) citer la source des informations qu'il ne peut pas attester personnellement

3) montrer les limites de ses connaissances

4) présenter ses conjectures comme des conjectures

5) ne jamais lire dans la tête des autres personnages

La première règle est facilement respectée car en remplaçant le «je qui raconte » par le «je qui écrit », Binet fait de l'histoire de l'attentat «une affaire personnelle » (p. 146). Par conséquent, l'auteur dans le rôle du narrateur parle de lui-même et des problèmes qu'il doit résoudre en essayant de marier deux phénomènes qu'il juge inconciliables : l'Histoire et la littérature, la fiction et la réalité. Bien conscient du fait que la fiction ne peut créer que l'illusion de la réalité, il décide de reposer son roman autant sur la création de cette illusion que sur son dévoilement constant :

Mais si je couche cette image sur le papier, comme je suis sournoisement en train de le faire, je ne suis pas sûr de lui rendre hommage. Je réduis cet homme au rang de vulgaire personnage, et ses actes à de la littérature : alchimie infamante mais qu'y puis-je ? Je ne veux pas traîner cette vision toute ma vie sans avoir, au moins, essayé de la restituer. J'espère simplement que derrière l'épaisse couche réfléchissante d'idéalisation que je vais appliquer à cette histoire fabuleuse, le miroir sans tain de la réalité historique se laissera encore traverser. (p. 10 $)^{5}$

Au moment où le narrateur ne peut plus se porter garant de la véracité des événements racontés, il indique la source de ses informations: témoignages, émissions, affiches et autres documents d'époque, catalogues d'expositions, ouvrages historiographiques et biographiques ainsi que toute une variété d'œuvres de fiction. Leur nombre respectable ainsi que le regard critique auquel il les soumet augmente considérablement la fiabilité du narrateur. Néanmoins, à cause de la mise en doute de la crédibilité de certains documents, les motifs qu'ils introduisent ne peuvent pas être authentifiés :

Je tombe sur le témoignage d'Helmut Knochen, nommé par Heydrich chef des polices allemandes en France, lors du passage de celui-ci à Paris. Il prétend révéler une confidence que lui fit Heydrich à cette occasion, et qu'il n'avait encore jamais répétée à personne. Ce témoignage date de... juin 2000, cinquante-huit ans plus tard ! Heydrich lui aurait dit : « La guerre ne peut plus être gagnée, il faudra trouver une paix de compromis et je crains qu'Hitler ne puisse l'admettre. Il faut y réfléchir. » Cette réflexion lui aurait donc été faite en mai 1942,

${ }^{5}$ C'est nous qui soulignons dans cet extrait aussi bien que dans les extraits suivants. 
avant Stalingrad, alors que le Reich n'a jamais semblé aussi fort ! [...] Pour ma part, je pense que c'est n'importe quoi. Mais je rapporte quand même. (p. 325-6)

Plus ou moins le même effet est obtenu quand le narrateur montre les limites de ses connaissances. D'une part, il augmente sa fiabilité quand il avoue ce qu'il ne sait pas, quand il se corrige, quand il précise ce qu'il aimerait savoir; d'autre part, il introduit beaucoup de motifs qu'il refuse d'authentifier :

Je suis absolument convaincu que si j'avais pu être dans sa tête à cet instant précis j'aurais eu de quoi raconter pour des centaines de pages. Mais je n'étais pas dans sa tête et je n'ai pas la moindre idée de ce qu'il a ressenti, je ne pourrais même pas trouver, dans ma petite vie, une circonstance qui m'aurait fait approcher d'un sentiment, même très dégradé, ressemblant à celui qui l'a envahi à cet instant. De la surprise, de la peur, avec un torrent d'adrénaline qui déferle dans les veines comme si toutes les vannes de son corps s'étaient ouvertes en même temps. (p 345)

Semblablement, le narrateur est assez strict dans la présentation de ses conjectures, hypothèses, incertitudes et doutes et le texte fourmille de probablement, peut-être, vraisemblablement, semble-t-il, il est possible, j'en doute, je ne sais pas exactement, si j'ose dire, je suppose, je présume, j'imagine, je parie, je n'exclus pas, je crois ou je veux croire. Le cas des hypothèses est particulièrement intéressant car le narrateur souvent oppose plusieurs motifs dont aucun n'est authentifié et qui, de plus, s'excluent respectivement :

Il offre le bouquet de violettes à Mme Zelenka et emprunte un imper, puis il repart. Ou bien il emprunte l'imper chez les Svatoš, qui avaient déjà prêté leur serviette, laissée elle aussi dans le virage, mais les Svatoš habitent plus loin, au cœur de la ville, près de la place Wenceslas ; ici, les témoignages ne sont pas clairs et je m'y perds un peu. Quoi qu'il en soit, il se rend ensuite au domicile des Fafek. (p. 367)

Parfois, le lecteur peut se sentir comme dans le jardin de Borges où les sentiers bifurquent sans arrêt tout en créant une série de mondes parallèles et de ce fait impossibles.

Finalement, pour établir son autorité performative, le narrateur personnel n'est pas censé lire dans les pensées des autres personnages. S'il veut rendre compte de leur état psychique, il doit se contenter de présomptions. En général, le narrateur de $H H h H$ respecte cette règle comme par exemple quand il déclare d'une manière ostentatoire : «Je ne suis pas Gabčík et je ne le serai jamais. Je résiste in extremis à la tentation du monologue intérieur et, ce faisant, me sauve peut-être du ridicule en cet instant décisif. » (p. 414)

Néanmoins, il est possible de dépister dans le roman plusieurs passages où le narrateur effectivement n'arrive pas à résister à cette tentation. Souvent il le signale au lecteur en épousant le rôle du «menteur avoué ». Ainsi, il décrit les pensées, les émotions et les sensations des protagonistes, transcrit les dialogues et expose des scènes entières, pour indiquer, après coup, la part de l'imagination :

Cette scène est parfaitement crédible et totalement fictive, comme la précédente. Quelle impudence de marionnettiser un homme mort depuis longtemps, incapable de se défendre! De lui faire boire du thé alors que si ça se trouve, il n'aimait que le café. De lui faire enfiler deux manteaux alors qu'il n'en avait peut-être qu'un seul à se mettre. De lui faire prendre le bus alors 
qu'il a pu prendre le train. De décider qu'il est parti un soir, et non un matin. J'ai honte. (p. 1445)

$\mathrm{Au}$ fur et à mesure que le roman avance, l'imagination et le romanesque s'introduisent de plus en plus dans le récit et, qui plus est, sans être indiqués par le narrateur. Tout d'abord, il s'agit de détails, ensuite des passages entiers qui, poétiques et romancés, font entrer le lecteur à Auschwitz sous le chant des corbeaux, à Košice où la neige tenace crisse sous les bottes ou à Prague où le grincement métallique du tramway encadre les réflexions de Gabčík. Plusieurs chapitres sont rédigés d'une manière très suggestive à la deuxième personne du pluriel pour mettre le lecteur dans la peau des parachutistes qui se préparent pour l'opération : "Vous êtes Jozef Gabčík ou Jan Kubiš, et vous allez entrer dans l'Histoire. » (p. 217) ou d'Heydrich lors de l'attentat : « Vous êtes fort, vous êtes puissant, vous êtes content de vous. Vous avez tué des gens, et vous allez en tuer beaucoup, beaucoup d'autres. Tout vous réussit. Rien ne vous résiste. En l'espace d'à peine dix ans, vous êtes devenu "l'homme le plus dangereux du III ${ }^{e}$ Reich" » (p. 342). Enfin, il y a le chapitre problématique où les parachutistes apprennent la destruction de Lidice. Conformément à ce qu'il vient de déclarer dans le chapitre précédent, le narrateur ne peut pas savoir ce qui se passait exactement dans la crypte et encore moins ce à quoi pensaient les parachutistes. Et pourtant, il écrit :

La nouvelle de la destruction du village plonge les deux parachutistes dans l'horreur et le désespoir. Plus que jamais, la culpabilité les ronge. Ils ont beau se dire qu'ils ont rempli leur mission, que la bête est morte, qu'ils ont débarrassé la Tchécoslovaquie et le monde de l'une de ses créatures les plus maléfiques, ils ont l'impression d'avoir eux-mêmes tué les habitants de Lidice, et aussi que tant qu'Hitler ne les saura pas morts, les représailles continueront indéfiniment. Enfermés dans leur crypte, ils ressassent tout ça dans leurs pauvres têtes brisées par la tension nerveuse et parviennent à la seule conclusion possible : il faut se rendre. Leur cerveau en feu imagine un scénario délirant : ils vont aller demander à être reçus par Emanuel Moravec, le Laval tchèque. (p. 401-2)

Il y a bien évidemment les aveux des premiers chapitres où le narrateur reconnaît qu'il aura recours à la stylisation : «Je prends donc le parti de styliser quelque peu mon histoire. » (p. 31) ; et, même, à l'invention : «Quoi qu'il en soit, mes dialogues, s'ils ne peuvent se fonder sur des sources précises, fiables, exactes au mot près, seront inventés. » (p. 33) Et Binet lui-même en parle ouvertement dans l'entrevue accordée à Olivia STRANSKY quand il estime la part de l'invention à 15 pour cent du livre entier (2014). Mais son comportement n'est pas absolument innocent. Déjà les motifs inventés qui deviennent plus importants vers la fin du livre montrent que l'auteur travaille de façon systématique et consciente. Ajoutons le fait qu'au début du roman, le narrateur signale d'une manière obsédante tous les passages imaginés ou inventés. Le lecteur, ainsi rassuré qu'il peut lui faire confiance, se trouve dans une situation difficile au moment où il réalise que cette obsession du narrateur a considérablement faibli. En fait, l'auteur n'est pas aussi sincère et son narrateur aussi fiable qu'ils veulent nous le faire croire. C'est comme si Binet voulait faire se perdre le lecteur dans le dédale construit à partir des éléments réels et imaginés. Au début, il lui laisse le choix. Quand le narrateur rapporte un témoignage qui ne lui paraît pas assez 
vraisemblable, il offre sa propre vision des choses et improvise un dialogue parfaitement inventé mais «un peu plus réaliste, un peu plus vivant, et probablement plus proche de la vérité. » (p. 165). Ici encore, il propose au lecteur de choisir la version du témoin direct bien qu'elle puisse être déformée. Mais peu après, il revient au choix difficile entre la vérité historique et l'invention romanesque. À l'instar de Valéry, il déclare vouloir éviter les phrases du type « la Marquise sortit à cinq heures » qui n'ont d'autre intérêt que « donner au texte la couleur du roman » (p. 176) et c'est effectivement ce qu'il finit par faire, tout intentionnellement, quand il réintroduit dans son texte la phrase : «Le sang lui monte aux joues, et il sent son cerveau gonfler dans sa boîte crânienne. » (p. 178) Non seulement le romanesque peut être plus près de la vérité, mais la vérité historique ne peut plus s'en passer. Et il paraît que le narrateur, lui aussi, finit par confondre les deux. Non seulement il souligne la dimension romanesque d'Heydrich et de toute l'histoire de l'attentat, non seulement il compare une séquence de l'attentat à une scène surréelle, non seulement il assimile la fuite de Gabčík à un cauchemar de Kafka, il se déplace en personne à Prague, « là, ici, en cette matinée claire du mercredi 27 mai $1942 »$ (p. 353). Il est sur place avec les parachutistes et il les regarde faire. Ensuite, dans la crypte, son «je » se confond avec eux pour former un «nous » collectif dans une tentative insensée d'échapper aux poursuivants : «Peut-être après tout y a-t-il un moyen, peut-être, si nous pouvons creuser dans la pierre. » (p. 425) Et finalement, le temps qui s'éternise efface la limite entre le présent du narrateur et celui des parachutistes :

Aujourd'hui nous sommes le 27 mai 2008. Quand les pompiers arrivent, vers 8 heures, ils voient des SS partout [...].

28 mai 2008. Les pompiers parviennent à glisser leur lance à incendie dans l'orifice de la meurtrière. [...]

29 mai 2008. L'eau commence à monter. [...]

30 mai 2008. L'eau monte un peu mais très lentement. (p. 426-8)

Il ne peut plus nous surprendre quand le narrateur se demande : «Pourquoi ai-je inventé cette phrase ? » et qu'il y répond paradoxalement: «Sans doute parce qu'il l'a vraiment prononcée. » (p. 378) Est-ce la fiction qui finit par imposer son image à la réalité ? Ou la réalité qui prédétermine la fiction ? C'est au lecteur de décider et peut-être aussi de trouver la sortie dudit dédale. C'est que Binet aime lire les auteurs qui savent jouer avec le lecteur (SEXTON, 2017) et il paraît que c'est effectivement ce qu'il fait lui-même dans son premier roman.

Outre le jeu avec le lecteur, l'auteur joue aussi avec la nature du monde fictionnel qu'il construit par son texte. Le narrateur dans le rôle du menteur avoué, la critique des sources, la mise en cause de la véracité des motifs ou même leur dénégation privent d'authentification un grand nombre de motifs concrets et donnent naissance aux mondes virtuels qui semblent coexister avec le monde fictionnel tout en le complétant ou le contredisant. De surcroît, l'autoreprésentation du récit dévoile son fonctionnement et sa nature fictionnelle ce qui, d'après DOLEŽEL, prive l'acte performatif de son pouvoir d'authentification car la création de l'illusion est doublée de la mise à nu de cette illusion. Par conséquent, le monde construit par une telle fiction reste virtuel : son 
existence est possible mais elle n'est pas attestée (2003, 163-4). Il est à noter que, même dans ce cas, l'auteur doit être conscient de sa pratique puisqu'elle permet de résoudre le dilemme principal de son narrateur : comment traiter l'Histoire s'il ne veut pencher ni du côté d'un «manuel d'histoire» (p. 146) embêtant, ni du côté d'un roman historique où la fiction l'emporte toujours sur l'Histoire. "Pour que quoi que ce soit pénètre dans la mémoire, il faut d'abord le transformer en littérature » (p. 244), dit-il. Mais comment le faire sans trahir l'Histoire ? Il n'y a qu'une seule possibilité : recourir à la ruse annoncée par l'épigraphe de la première partie : "À nouveau la pensée du prosateur fait des taches sur l'arbre de l'Histoire, mais ce n'est pas à nous de trouver la ruse qui permettrait de faire rentrer l'animal dans sa cage portative. (Ossip Mandelstam, "La Fin du roman") $»^{6}$. La ruse dont Binet se sert consiste effectivement dans la simulation de la crédibilité du narrateur dont le mensonge dissimule le caractère virtuel du monde qu'il construit. Par son refus d'authentifier le monde construit par le narrateur, l'auteur enlève l'existence fictionnelle à toutes les entités, y compris les personnages qui n'habitent qu'un monde virtuel, flottant quelque part entre l'être et le non-être: «Les gens qui ont participé à cette histoire ne sont pas des personnages ou en tout cas s'ils le sont devenus par ma faute, je ne souhaite pas les traiter comme tels. » (p. 433) C'est sous cette lumière qu'il faut comprendre le dernier chapitre du roman où tous les personnages apparaissent comme des fantômes à bord d'un paquebot qui «glisse sur la Baltique comme un poème de Nezval. » (p. 442) Il y a Gabčík et Kubiš et «d'autres fantômes de l'armée tchécoslovaque » (p. 442), les ombres des vieillards, des dames seules et des enfants sages et il y a aussi une jeune femme qui ressemble à Natacha, la copine du narrateur, et finalement peut-être le narrateur lui-même.

La lutte entre la réalité et la fiction que Binet mène dans $H H h H$ lui permet d'avoir la conscience nette, de regarder ses héros, ces personnages qui ont « vraiment existé » (p. 9), droit dans les yeux tout en laissant son narrateur crier sans cesse, un peu à la manière de René Magritte, que ce qu'il écrit n'est pas un roman historique. Et il a raison car ceci n'est pas un roman historique mais un roman sur la représentation de l'Histoire. Dans son œuvre postmoderne Binet réagit à la modernité des auteurs qui refusent de créer l'illusion de la réalité. Non seulement il est conscient que la fiction ne peut que simuler la réalité, c'est effectivement cette conscience qui lui permet finalement de raconter l'Histoire, de la transformer en histoire.

Mais Binet ne s'arrête pas là. La pratique des dialogues inventés et placés dans un contexte réel se trouve, inversée, dans La septième fonction du langage où les citations des grands penseurs se voient transformées en dialogues et placées dans un contexte imaginé. Semblablement, si Binet prive les personnages de $H H h H$ de leur existence, il procure à Simon Herzog de La septième fonction le doute sur la nature réelle de son existence. Mais le doute, ne fonde-t-il pas l'existence ? Si le narrateur de $\mathrm{HHhH}$ ment avec scrupules, celui de La septième fonction le fait tout

\footnotetext{
${ }^{6}$ L'importance de la ruse est confirmée par le choix de l'épigraphe pour le second roman de Binet : « II y a des interprètes partout. Chacun parle sa langue même s'il connaît un peu la langue de l'autre. Les ruses de l'interprète ont un champ très ouvert et il n'oublie pas ses intérêts. (DERRIDA) »
} 
franchement, ouvertement et sans scrupules. La position antithétique de ces deux méta-romans ne peut que confirmer une pratique consciente et systématique de Laurent Binet qui, dans son premier roman, manipule délibérément le lecteur tout en jouant avec sa confiance ainsi qu'avec la sincérité du narrateur-auteur. Et c'est effectivement cette intentionnalité ensemble avec le travail systématique de l'auteur qui font preuve que sa sincérité n'a rien de sincère, elle est en réalité consciemment fabriquée et avec elle l'acte d'énonciation. Mais si l'acte d'énonciation est fabriqué, s'il n'est pas sincère, n'est-il pas aussi simulé ?

\section{BIBLIOGRAPHIE}

Austin J. L. (1962), How to do things with words, Oxford, The Clarendon Press. BIRNBAUM Jean (2015), «Les Incorruptibles », version Laurent Binet, Le Monde, le 19 août 2015. Disponible sur : http://www.lemonde.fr/livres/article/2015/08/19/les-incorruptibles-versionlaurent-binet_4730280_3260.html (consulté le 10 mars 2017).

BORDERIE Laurent (2015), Laurent Binet et le polar sémiologique, L'orient littéraire, 2015-09, numéro 111. Disponible sur: http://www.lorientlitteraire.com/article_details.php?cid=31\&nid=6241 (consulté le 10 mars 2017).

DolEŽEL Lubomír (2003), Heterocosmica: fikce a možné světy, Praha, Karolinum.

HAMOUCHI Maïté (2015), Pourquoi tuer Rolan Barthes ?, Metro, le 6 novembre 2015. Disponible sur : https://fr.metrotime.be/2015/11/06/interview/pourquoituer-rolan-barthes/\# (consulté le 10 mars 2017).

LEMÉNAGER Grégoire (2015), Roland Barthes a-t-il été assassiné ? L'Obs, le 22 août 2015. Disponible sur : http://bibliobs.nouvelobs.com/romans/20150821.OBS4517/laurent-binetroland-barthes-assassine.html (consulté le 10 mars 2017).

LEVISALLES Natalie (2015), Barthes selon Binet : «la science de Sherlock Holmes », Libération, le 4 septembre 2015. Disponible sur: http://next.liberation.fr/livres/2015/09/04/barthes-selon-binet-la-science-desherlock-holmes_1375873 (consulté le 17 mars 2017).

LEZARD Nicholas (2017), Teasing the French academy to death, The Spectator, 13 May 2017. Disponible sur : https://www.spectator.co.uk/2017/05/teasing-thefrench-academy-to-death/\# (consulté le 20 mars).

LIGER Baptiste (2015), Laurent Binet signe un roman complotiste aussi jubilatoire que documenté, L'Express, le 12 septembre 2015. Disponible sur: http://www.lexpress.fr/culture/livre/laurent-binet-signe-un-roman-complotisteaussi-jubilatoire-que-documente_1708599.html (consulté le 10 mars 2017).

PHILIPPE Elisabeth (2015a), C'est Barthes qu' on assassine, Les Inrockuptibles, le 19 août 2015. Disponible sur: http://www.pressreader.com/france/lesinrockuptibles/20150819/282243779332511 (consulté le 10 mars 2017).

PHILIPPE Elisabeth (2015b), Laurent Binet, prix Interallié : “Le langage, c'est le pouvoir", Les InRocks, le Wednesday 19 August 2015. Disponible sur: 
http://mobile.lesinrocks.com/2015/08/19/livres/laurent-binet-le-langage-cestle-pouvoir-11767933/ (consulté le 15 mars 2017).

SAMOYAUlT Tiphaine (2015), Adhésion in "Qui a tué Roland Barthes? Le polar de la rentrée », Philippe Sollers : sur et autour de Sollers, le 21 août 2015. Disponible sur: http://www.pileface.com/sollers/spip.php?article1633 (consulté le 10 mars 2017).

SARFATI Sonia (2015), Laurent Binet: le langage est son arme, La Presse, le 9 octobre 2015.

Disponible sur : http://www.lapresse.ca/arts/livres/entrevues/201510/09/01-4908327-laurentbinet-le-langage-est-son-arme.php (consulté le 17 mars 2017).

SEARLE John (2007), Logický status fikčního diskurzu, Aluze 1/2007, p. 61-69. Disponible sur : http://aluze.cz/2007_01/07_Studie_-_Searle.php (consulté le 15 mars 2017).

SEXTON David (2017) The 7th Function of Language by Laurent Binet, EveningStandard, Thursday 27 April 2017. Disponible sur: http://www.standard.co.uk/lifestyle/books/the-7th-function-of-language-bylaurent-binet-review-a3525626.html (consulté le 20 mars 2017).

STRANSKY Olivia (2014), «I Enjoy Correcting Myself»: An Interview with Author Laurent Binet, Sampsonia Way Magazine, le 18 mars 2014. Disponible sur: $\quad$ http://www.sampsoniaway.org/interviews/2014/03/18/\%E2\%80\%9Cienjoy-correcting-myself\%E2\%80\%9D-an-interview-with-author-laurent-binet/ (consulté le 16 mars 2017).

TREINER Sandrine (2015), Laurent Binet, La septième fonction du langage, France Culture, le 21 août 2015. Disponible sur : https://www.franceculture.fr/emissions/les-bonnes-feuilles/laurent-binet-laseptieme-fonction-du-langage (consulté le 20 mars 2017). 